\section{P083 DESIGNING ONLINE HIV INFORMATION, TESTING AND CLINICAL CARE PATHWAYS TO ADDRESS USERS' EXPECTATIONS AND EMOTIONS}

${ }^{1}$ Jo Gibbs*, ${ }^{2}$ Aneesha Singh, ${ }^{3}$ Claudia Estcourt, ${ }^{1}$ Pam Sonnenberg, ${ }^{4}$ Ann Blandford. ${ }^{1}$ University College London, Centre for Population Research in Sexual Health and HIV, Institute for Global Health, London, UK; ${ }^{2}$ University College London, UCL Interaction Centre, London, UK; ${ }^{3}$ Glasgow Caledonian University, School of Health and Life Sciences, Glasgow, UK; ${ }^{4}$ University College London, Institute of Digital Health, London, UK

10.1136/sextrans-2019-sti.278

Background Advances in STI/HIV diagnostics and digital health enable people to self-sample, self-test and receive results remotely. However, concerns remain about poor support and linkage to care. We explored how people access online HIV resources and how technology can facilitate testing, to inform development of software linking those with reactive results to care, and those with negative results to testing/retesting for HIV and other STIs, health promotion and biomedical interventions (e.g. PrEP).

Methods A prototype was developed following the eClinical Care Pathway Framework which consists of an interdisciplinary 9-step approach. A convenience sample of people $(\mathrm{n}=28$, aged 25-60, 36\% female, 57\% MSM, ethnicallydiverse, 6 people living with HIV) participated in semi-structured interviews and 'think-aloud' exercises exploring the following user journey using existing online and remote testing resources: contemplating testing $\rightarrow$ searching for information online $\rightarrow$ choosing how to test $\rightarrow$ ordering a test $\rightarrow$ experiences using HIV self-sampling/self-testing $\rightarrow$ receiving a result $\rightarrow$ engaging with care. The content and sequence of a paper prototype HIV online results service and negative/reactive pathways were cognitively tested. Findings informed the design of a software prototype which was iteratively developed with further user testing. Qualitative data were analysed thematically.

Results Existing HIV online and remote testing resources failed to 1) meet participants' needs and expectations related to information provision/support and 2) address anxieties related to testing. Participants expressed strong emotions about the wording and sequence of text with negative/reactive pathways, and highlighted the need for the resource to provide a rationale for collecting sensitive data. Adjusting the way in which information was provided and reactive results were presented attenuated, but did not remove, associated negative emotions. Some participants felt people choosing self-sampling should not receive reactive results online.

Conclusion Tailoring information, careful wording, and clear messaging and signposting throughout online HIV testing/diagnosis care pathways are essential to address expectations, attenuate strong emotional reactions, and support linkage and engagement with care.

Disclosure No significant relationships.

\section{P084 GEOGRAPHICAL PROXIMITY AS BARRIER IN SEXUAL HEALTH CLINIC UTILIZATION, EVEN IN INFRASTRUCTURE RICH COUNTRIES}

${ }^{1}$ Denise Twisk*, 'Bram Meima, ${ }^{2}$ Hannelore Götz. 'Municipality of Rotterdam, Research and Business Intelligence, Rotterdam, Netherlands; ${ }^{2}$ Public Health Service Rotterdam Rijnmond, Public Health/Sexual Health, Rotterdam, Netherlands

\subsection{6/sextrans-2019-sti.279}

Background The greater Rotterdam area of the Netherlands consists of 15 municipalities. The sole center of sexual health $(\mathrm{CSH})$ plays a crucial role in finding people unaware of their STI/HIV status. We investigated the association between $\mathrm{CSH}$ utilization and sociodemographic and geographical factors. The primary focus was on geographical proximity.

Methods We linked CSH consultation data from STI tested heterosexual clients to the population registry for the years 2015-2017. In this analysis, logistic regression was performed to investigate the importance of proximity. We stratified by age (15-24 and 25-44 years) and ethnicity, since CSH access policies may affect utilization. Proximity was operationalised as straight-line distance between centroid address of a 4-digit postal code and CSH address. Distance was divided into six categories: $<10^{\text {th }}$ percentile $(<1.8 \mathrm{~km}), 10-25^{\text {th }}$ percentile $(1.8-2.9 \mathrm{~km}), 25-50^{\text {th }}$ percentile $(2.9-6.1 \mathrm{~km}), 50-75^{\text {th }}$ percentile $(6.1-9.9 \mathrm{~km}), 75-90^{\text {th }}$ percentile $(9.9-17.3 \mathrm{~km})$ and $>90^{\text {th }}$ percentile $(>17.3 \mathrm{~km})$.

Results In total, 19,287 out of the 1.5 million inhabitants aged 15-44 utilized the CSH in the study period. Our data suggest that larger distance to the $\mathrm{CSH}$ is strongly associated with lower utilization. For instance, the odds ratios for utilization by 15-24-year-old Surinamese, adjusted for sex, were $0.70 \quad(95 \%$ CI: $0.59-0.84), 0.61 \quad(0.52-0.71), 0.52 \quad(0.44$ $0.63), 0.34(0.28-0.45)$ and $0.23(0.13-0.43)$ for the different percentiles respectively, compared to $<10^{\text {th }}$ percentile (overall $\mathrm{P}<0.001)$. Although the association was observed for all ethnic groups, including native Dutch, the strength of the association varied by ethnic group.

Conclusion Geographical proximity effects healthcare utilization even in an infrastructure rich country. The extent to which GPs compensate for lower CSH utilization for those further away from the CSH is unclear. Further research will address the role of the GP in STI healthcare to develop an optimal strategy for offering local STI testing services.

Disclosure No significant relationships. 